\title{
The Effect of Early Poststressor Intervention with Sertraline on Behavioral Responses in an Animal Model of Post-Traumatic Stress Disorder
}

\author{
Michael A Matar', Hagit Cohen*,', Zeev Kaplan' and Joseph Zohar ${ }^{2}$ \\ 'Anxiety and Stress Research Unit, Ministry of Health Mental Health Center, Faculty of Health Sciences, Ben-Gurion University of the Negev, \\ Beer-Sheva, Israel; ${ }^{2}$ The Chaim Sheba Medical Center, Sackler Medical School, Tel-Aviv University, Tel-Aviv, Israel
}

\begin{abstract}
Whereas several well-controlled studies have established the selective serotonin reuptake inhibitors (SSRIs) as the recommended firstline pharmacotherapeutic agents for acute and chronic post-traumatic stress disorder (PTSD), drug interventions in the acute postexposure phase have not been studied to the same extent and tend to be largely speculative. This study employed an animal model which assesses prevalence of individual stress-response behavior patterns in order to assess the short-term effects of a brief treatment regimen with an SSRI (sertraline) administered immediately after stress-exposure, with those of an identical delayed regimen and of saline. Prevalence rates of rats displaying extreme anxiety-like behavioral responses to predator stress, compared to partial and minimal responses, were assessed in the elevated plus maze and startle response paradigms, with and without intraperitoneal administration of sertraline for 7 days immediately postexposure, or 7 days after exposure. Immediate postexposure administration of sertraline reduced anxiety-like and avoidant behavior, decreased hyperarousal responses and diminished the overall incidence of extreme (PTSD-like) behavioral responses, compared to the delayed treatment regimen and to saline controls. Brief immediate poststress exposure treatment with sertraline reduced prevalence rates of extreme behavioral disruption in the short-term. SSRI drugs are thus worthy of further investigation as agents of secondary prevention in the acute aftermath of stress-exposure.

Neuropsychopharmacology (2006) 3 I, 2610-2618. doi: I0.1038/sj.npp. I 30 I I32; published online 21 June 2006
\end{abstract}

Keywords: post-traumatic stress disorder; animal model; selective serotonin reuptake inhibitors; extreme behavioral response; early intervention; secondary prevention

\section{INTRODUCTION}

Post-traumatic stress disorder (PTSD) is an incapacitating chronic syndrome reflecting a disorder of cognitive, emotional, and physiological processing and/or recovery from the initial reaction to exposure to a potentially traumatic experience.

Whereas several well-controlled studies have established the selective serotonin reuptake inhibitors (SSRIs) as the recommended first-line pharmacotherapeutic agents for acute and chronic PTSD (Davidson, 2003; Davidson, 2004a; Tucker et al, 2004; Zohar et al, 2002), drug interventions in the acute phase (up to 1 month postexposure) have not been studied to the same extent and tend to be largely speculative

*Correspondence: Dr H Cohen, Anxiety and Stress Research Unit, Ministry of Health Mental Health Center, Faculty of Health Sciences, Ben-Gurion University of the Negev, PO Box 4600, Beer-Sheva 84 170 , Israel, Tel: +97286401743, Fax: +97286401742,

E-mail: hagitc@bgu.ac.il

Received 8 November 2005; revised 8 May 2006; accepted 12 May 2006

Online publication: 23 May 2006 at http://www.acnp.org/citations/ Npp052306050669/default.pdf
(Bryant, 2005; Pitman and Delahanty, 2005; Shalev, 2002). Most existing treatment guidelines for PTSD point to judicious use of the same group of medications, although they specify that this suggestion is based on sparse empirical data and represents an extrapolation of PTSD practice guidelines, based on common practice (Foa $e t$ al, 2005; Stein et al, 2003; Ursano et al, 2004; Weine et al, 2002).

The study of adrenergic drugs as agents of secondary prevention by Pitman et al (2002) is worthy of mention. The authors reported that the prevention of presynaptic norepinephrine release with $\alpha$-2-adrenergic agonists, or the blockade of postsynaptic norepinephrine receptors by $\beta$-adrenergic antagonists such as propranolol, reduce hormonally enhanced memories and fear conditioning, and have potentially therapeutic effects on the clinical course of subsequent PTSD symptoms. Similar studies of other therapeutic agents are needed.

An animal model of PTSD which intentionally classifies the exposed population into groups according to individual response patterns is particularly advantageous in complementing clinical studies of early postexposure drug interventions. In this animal model populations of exposed rodents are classified according to the degree of their 
individual behavioral response using standardized 'cutoff behavioral criteria' (CBC), creating three distinct groups entitled 'extreme behavioral responses' (EBR) and 'minimal behavioral response' (MBR) at the extremes, and a middle group of partial behavioral response (PBR) (Cohen et al, 2003, 2004, 2005, 2006a, 2006b; Cohen and Zohar, 2004). Primarily, the advantage lies in the controlled prospective design that animal models enable and since response patterns are the focus of the model employed, the prevalence rates of individuals displaying extremely disrupted postexposure behavior can serve to provide an initial indication of the effect of the drug under study. Once this basic effect has been confirmed or refuted, the next step would be to examine the effect on long-term 'morbidity', that is, prevalence of PTSD-like behaviors.

This study thus aimed to provide preliminary data regarding the effect on prevalence rates of individuals displaying extreme, partial, and MBR to predator scent stress (PSS) in a group of animals treated briefly with an FDA-approved SSRI treatment for PTSD — sertraline - for 1 week immediately after exposure, in comparison with a group given the same regimen at a later time, each compared to placebo-treated controls and to unexposed controls.

The working hypothesis was that early intervention with sertraline would reduce the prevalence rates of extreme responders and increase the prevalence of partial and/or minimal responders at least as effectively as the delayed treatment regimen.

\section{MATERIALS AND METHODS}

All procedures were carried out under strict compliance with ethical principles and guidelines of the NIH Guide for the Care and Use of Laboratory Animals. All treatment and testing procedures were approved by the Animal Care Committee of Ben-Gurion University of the Negev, Israel.

\section{Animals}

In total, 305 adult male Sprague-Dawley rats weighing 150$200 \mathrm{~g}$ were employed. Animals were habituated to the housing conditions for at least 10 days. During that time the rats were handled once daily, that is, picked up with a gloved hand. The animals were housed four per cage in a vivarium with stable temperature and a reversed 12-h light/ dark cycle, with unlimited access to food and water. All testing was performed during the dark phase under dim light conditions.

The experiment was carried out in two parts: immediate treatment and delayed treatment. In all, 237 rats participated in study 1 , and 68 in study 2 . In each part of the experiment, behaviors were assessed in the elevated plus maze (EPM) and the acoustic startle response (ASR) tests.

\section{Predator Scent Stress}

The stress paradigm consisted of placing the test animals on well-soiled cat litter for $10 \mathrm{~min}$ (in use by the cat for 2 days, sifted for stools). The control animals were exposed to fresh, unused litter for the same amount of time.

\section{Drug}

Rats in the treatment groups were given sertraline hydrochloride (Pfizer, Israel) $10 \mathrm{mg} / \mathrm{kg} / \mathrm{day}$, injected once daily intraperitoneally (i.p.) for 7 days. The dose is based on that used previously by Marona-Lewicka and Nichols (1997); Thomas et al (1998); Maj and Rogoz (1999) and Kim et al (2002). Control groups were given $0.9 \%$ saline solution in the same manner in order to control for the stressful effect of the i.p. administration route.

\section{Experimental Procedures}

Study 1: global effects of sertraline immediately after PSS exposure. Rats were randomly assigned into three groups: (1) Unexposed control animals: rats exposed to fresh, unused litter for $10 \mathrm{~min}$; (2) exposed + immediate sertraline (IS) animals: rats exposed to PSS for $10 \mathrm{~min}$, and $1 \mathrm{~h}$ after that given sertraline for 7 days; (3) exposed + immediate saline controls (IC) animals: rats exposed to PSS for $10 \mathrm{~min}$, and $1 \mathrm{~h}$ after that given saline for 7 days.

Behavioral reactions were tested $6 \mathrm{~h}$ after the last dose of sertraline. The assessment of rats seven days postexposure has previously been established by Adamec (1997), and has been shown to refer to long-term PTSD-like behaviors. Our previous results (Cohen et al, 2004) have repeatedly shown that as of day 7 , the prevalence rate of EBR remains constant at about 25\% until day 30. The DSM-IV defines PTSD as lasting more than a month after the exposure and thus the persistence of anxiety symptoms on day 7 after exposure may be taken to fulfill the time criterion of PTSD in rodents (Adamec, 1997).

Study 2: global effect of delayed treatment regimen on the entire population. Rats were randomly assigned into three groups: (1) Unexposed control animals: rats exposed to fresh, unused litter for $10 \mathrm{~min}$; (2) exposed + delayed sertraline (DS) animals: rats exposed to PSS and 7 days after that given sertraline for 7 days; (3) exposed + delayed saline control (DC) animals: rats exposed to PSS and 7 days after that given saline for 7 days.

Behavioral reactions were tested at completion of the treatment regimen, $6 \mathrm{~h}$ after the last dose of sertraline, that is, 14 days postexposure.

\section{Behavioral Paradigms}

The EPM. The maze consists of a plus-shaped platform with two open arms and two closed arms - surrounded by $14-\mathrm{cm}$ high opaque walls on three sides, with arms of the same type located opposite each other, leaving a $10 \mathrm{~cm}^{2}$ central area (File, 1993). Each rat was placed on the central platform facing an open arm and was allowed to explore the maze for $5 \mathrm{~min}$. Each test was videotaped and scored by an independent observer. Arm entry was defined as entering the arm with all four paws.

The behaviors assessed were: duration in open and closed arms, and on the central platform; open and closed arm entries; and total entries into all arms.

Acoustic startle response. Startle response was measured using two ventilated startle chambers (SR-LAB system, San Diego Instruments, San Diego, CA). Each chamber 
consisted of a Plexiglas cylinder resting on a platform inside a sound attenuated, ventilated chamber. Movement inside the tube was detected by a piezoelectric accelerometer below the frame. Sound levels within each test chamber were measured routinely using a sound level meter (Radio Shack) to ensure consistent presentation (SR-LAB system, San Diego Instruments, San Diego, CA). Chambers were calibrated daily. The animals were placed inside the tube and allowed a 5-min acclimatization period with background noise only. Following a $5 \mathrm{~min}$ acclimatization period, 30 acoustic startle trials $(110 \mathrm{~dB}$ white noise; $40 \mathrm{~ms}$ duration; 30 or $45 \mathrm{~s}$ intertrial interval) were presented over the $68 \mathrm{~dB}$ white noise background.

Two measures of behavior were assessed: (1) mean startle amplitude; (2) percent of startle responses habituation over repeated presentation of the acoustic pulse. Startle amplitude was averaged over all 30 stimuli. Percent habituation was calculated by comparing the responses in the first and last six stimulus blocks:

Percent habituation

$=100 \times[($ average startle amplitude in Block 1$)$

- (average startle amplitude in Block 5)]

/(average startle amplitude in Block 1$)$.

\section{Classification According to $\mathrm{CBCs}$}

The classification of individuals according to the degree to which behavior is affected by a stressor is based on the premise the extremely compromised behavior in response to the priming trigger is not conducive to survival and is thus maladaptive, representing a pathological degree of response. The procedure requires the following steps:

(a) Verification of global effect. The data must demonstrate that the stressor had a significant effect on the overall behavior of exposed $v s$ unexposed populations at the time of assessment.

(b) Application of the CBCs to the data. The CBCs used herein have been repeatedly validated in a large series of studies in rodents (Cohen et al, 2003, 2004, 2006a, 2006b; Cohen and Zohar, 2004) and in a mathematical model (Cohen et al, 2005).

Extremely compromised exploratory behavior on the plus-maze and markedly increased startle reaction that does not undergo habituation (defined below) were taken to reflect anxiety-like behaviors, that is, fearfulness and hypervigilance.

In order to maximize the resolution and minimize false positives, extreme responses to both of these paradigms performed in sequence were required for 'inclusion' into the EBR group (anxious, fearful, and hypervigilant that is PTSDlike symptoms), whereas a negligible degree of response to both was required for inclusion in the MBR group.

Definition of CBCs on each paradigm:

(a) $E B R$

(1) Five minutes (entire session) spent in closed arms and no entries into the open arms of the EPM.
(2) Mean amplitude of the startle response (at $110 \mathrm{~dB}$ ) $>1200$ units and startle response shows no habituation over time.

(b) $M B R$

(1) $0-1$ min spent in closed arms and $\geqslant 8$ open-arm entries on the EPM.

(2) Mean amplitude of the startle response (at $110 \mathrm{~dB}$ ) $<700$ units and habituation is demonstrated.

Prevalence rates for EBR and MBR are calculated from the overall data. PBR (individuals who quality for neither EBR nor MBR) are at present not further subdivided.

\section{Statistical Analyses}

For the ASR results and the EPM behaviors, the statistical analyses were performed using one-way analysis of variance (ANOVA). Where significant group effects were detected, Bonferroni test indicated significant post hoc differences between individual groups.

The prevalence of EBR, PBR, and MBR rats as a function of study group was tested using cross-tabulation and $\chi^{2}$-tests.

\section{RESULTS}

\section{Study 1: Global Effects of Sertraline Immediately after PSS Exposure}

Anxiety-like behaviors (EPM). As shown in Figure 1, there were significant differences between the groups in terms of the time spent in the open arms $(\mathrm{F}(2,234)=6.1$, $p<0.003$ ) (Figure 1a) and number of entries to the open arms $(\mathrm{F}(2,234)=7.0, p<0.0015)$ (Figure $1 \mathrm{~b})$. PSS exposure followed by administration of saline elicited significantly decreased overall time spent in the open arms of the maze, as compared to unexposed control conditions (post hoc Bonferroni test: $t=2.86, p<0.015)$. Injection of sertraline immediately after PSS exposure significantly corrected postexposure anxiety-like behavior relative to the data for animals treated with saline, resulting in a significant relative increase in total time spent in the open arms of the maze (post hoc Bonferroni test: $t=2.88, p<0.02$ ) (Figure 1a) and in number of entries to the open arms (post hoc Bonferroni test: $t=3.75, p<0.0007$ ) (Figure $1 \mathrm{~b}$ ). In effect, there were no significant differences between the sertraline-injected group and unexposed controls on these measures.

No differences were observed in total exploration on the maze between groups (Figure 1c), suggesting overall anxiety-like behavior and avoidance of exploration in the open arms, as opposed to a nonspecific impairment of locomotion, supporting the validity of the model (Adamec et al, 2003; Adamec and Shallow, 1993).

\section{ASR and Habituation}

Figure 2a shows the mean startle amplitude of rats in response to the 30 startle pulses. There were significant differences between the groups $(\mathrm{F}(2,234)=12.35$, $p<0.0001)$. Post hoc Bonferroni test revealed that PSS 

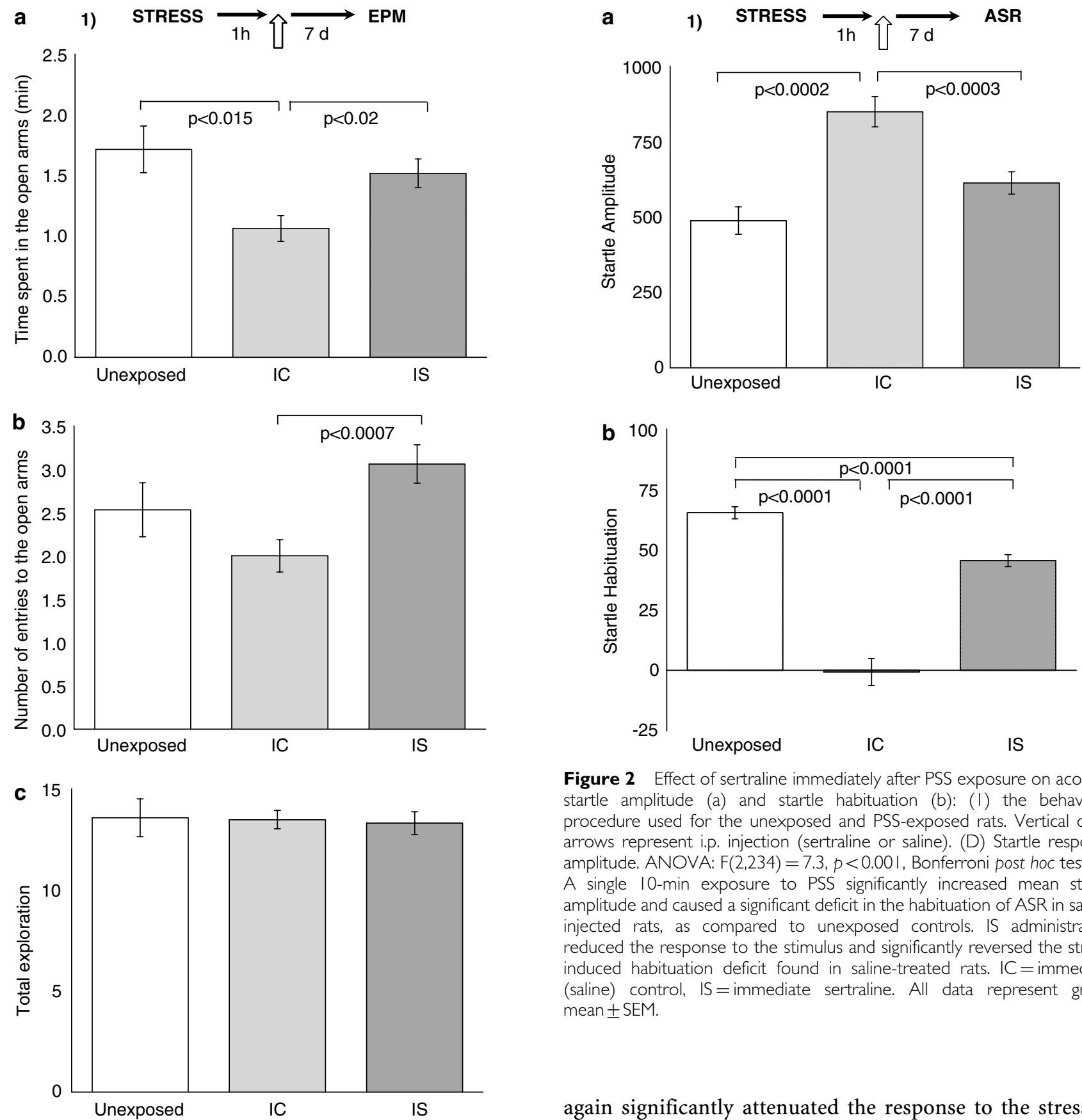

Figure I Effect of sertraline immediately after PSS exposure on anxietylike behaviors: (I) the behavioral procedure used for the unexposed and PSS-exposed rats. Vertical open arrows represent i.p. injection (sertraline or saline). (a) Time spent in the open arms of the EPM. ANOVA: $F(2,234)=6.1, p<0.003$, Bonferroni post hoc test: $I \neq=1$, III. (b) Number of entries to the open arms of the EMP. ANOVA: $F(2,234)=7.03, p<0.00$ I, Bonferroni post hoc test: $\|\neq\|$ I. (c) Total exploration on the EMP. ANOVA: NS. A single I0-min exposure to PSS followed by immediate administration of saline significantly increased anxiety-like responses to open spaces as compared to nonexposed control animals. IS administration significantly decreased the anxiety-like response. IC =immediate (saline) control, $I S=$ immediate sertraline. All data represent group mean \pm SEM.

exposure significantly increased the mean startle amplitude in exposed rats injected with saline, as compared to controls $(t=4.2, p<0.0002)$. Sertraline immediately after exposure

again significantly attenuated the response to the stressor, eliciting a relative decrease in postexposure mean startle amplitude compared to the saline-control group (post hoc Bonferroni test: $t=3.96, p<0.0003)$. There were no significant differences between the immediate postexposure sertraline group and the unexposed controls in terms of mean startle amplitude.

Figure $2 \mathrm{~b}$ shows the 'percent habituation'-parameter of the startle response in all groups. Percent habituation differed significantly among the groups $(\mathrm{F}(2,234)=153.4$, $p<0.0001)$. Post hoc Bonferroni test revealed that PSS exposure followed by saline caused a significant deficit in the habituation of the ASR in exposed animals compared with naïve rats $(t=14.3, p<0.0001)$ and compared to sertraline-treated animals $(t=14.4, p<0.0001)$. IS administration reversed the trend of the stress-induced habituation deficit to a significant degree, although unexposed rats 
exhibited significantly better habituation than sertralinetreated rats $(t=4.3, p<0.0001)$.

To summarize, immediate postexposure administration of sertraline corrected anxiety-like behaviors on the EPM and amplitude of ASR, and had a partial effect on habituation, relative to saline.

\section{Reanalysis Applying CBC}

There were significant differences in the prevalence rates of individuals displaying an extreme degree of behavioral responses (EBR) among groups (Pearson $\chi^{2}=15.7, \mathrm{df}=2$, $p<0.0004)$. As shown in Figure $3 \mathrm{a}$, there were no EBR individuals amongst the controls. The prevalence of EBR among PSS-exposed rats injected with saline was $30 \%$ of the total population $\left(\chi^{2}=12.88, p<0.0003\right)$. Injection of sertraline immediately after PSS exposure diminished the prevalence of EBR rats to $17 \%$ of the total population, a significant effect as compared to the saline treatment group $\left(\chi^{2}=5.47, p<0.02\right)$ and as compared to controls $\left(\left(\chi^{2}=16.18, p<0.015\right)\right.$.

There were no significant differences in the prevalence of minimally affected individuals (MBR) among groups (Figure 3b).

There were significant differences in the prevalence of individuals displaying PBR among groups (Pearson $\chi^{2}=9.35, \mathrm{df}=2, p<0.01$ ) (Figure 3c). The stringency of the criteria for MBR was validated by a prevalence rate of 90\% PBR in unexposed controls. PSS exposure elicited a drop in PBR prevalence rates to $62.5 \%$ in saline-treated controls $\left(\chi^{2}=9.1, p<0.003\right)$ and to lesser degree $(71.5 \%)$ in the IS group $\left(\chi^{2}=5.0, p<0.03\right)$.

\section{Study 2: Global Effect of the DS Regimen}

Anxiety-like behaviors (EPM). As shown in Figure 4, there were significant differences between the groups in terms of the time spent in the open arms $(\mathrm{F}(2,65)=9.7, p<0.0003)$ (Figure 4a) and number of entries to the open arms $(\mathrm{F}(2,65)=3.5, p<0.035)$ (Figure $4 \mathrm{~b})$. PSS exposure followed by the delayed 7-day treatment regimen with saline resulted in significantly decreased time spent in the open arms (Figure $4 \mathrm{a}$ ) and open arm entries (Figure $4 \mathrm{~b}$ ), as compared to unexposed controls (post hoc Bonferroni test: $t=2.95$, $p<0.015$; and $t=4.3, p<0.0002$, respectively). The DS regimen again significantly attenuated global postexposure anxiety-like behavior, resulting in a significant relative increase in total time spent in the open arms (Figure 4a) and in number of entries into the open arms (Figure $4 \mathrm{~b}$ ), relative to the response patterns of the saline treatment group (post hoc Bonferroni test: $t=2.5, p<0.05$; and $t=2.5, p<0.05$, respectively), so that there were no significant differences between the sertraline-injected group and the unexposed controls.

No differences in total exploration were observed between groups (Figure 4c).

\section{ASR and Habituation}

There were significant differences between the groups in startle amplitude $(\mathrm{F}(2,65)=13.8, p<0.0001)$. The exposedsaline treatment group displayed significantly increased
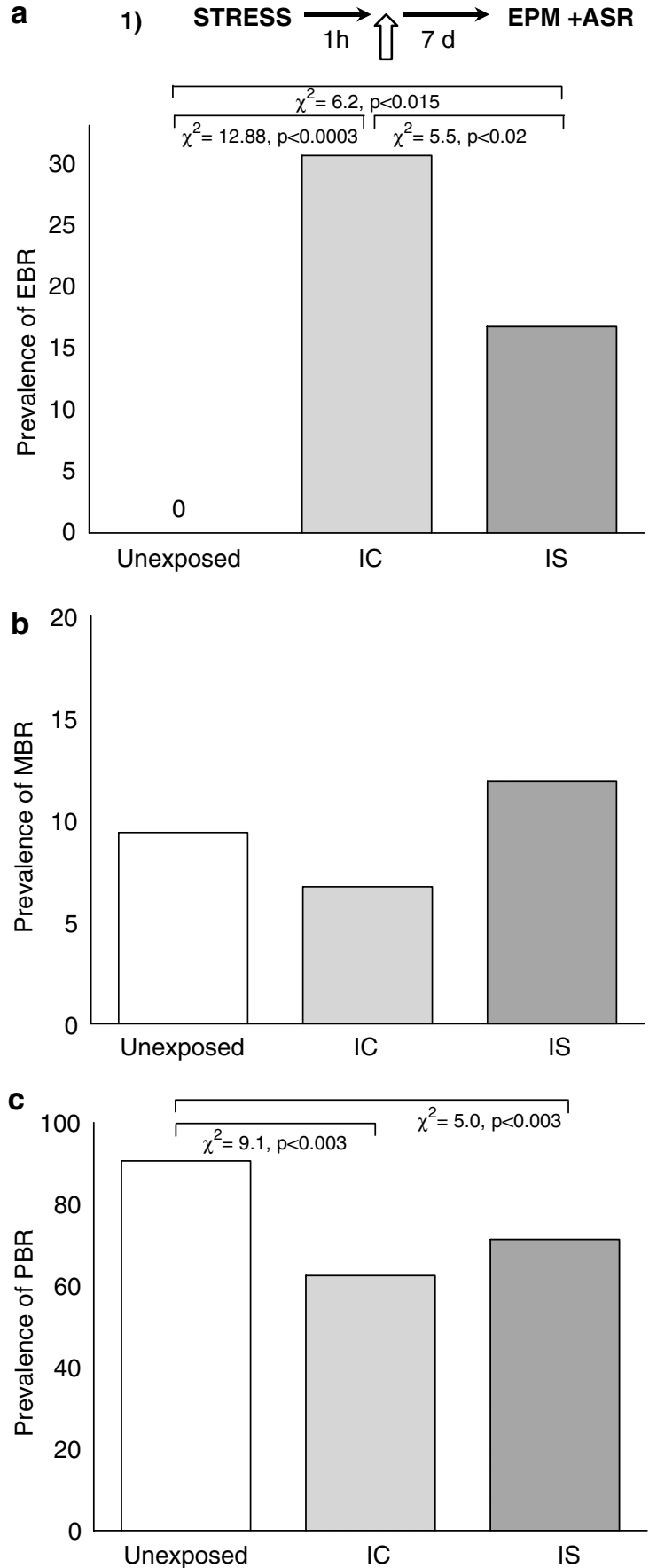

Figure 3 Effect of IS after PSS exposure on the prevalence of EBR vs PBR and MBR individuals: (I) the behavioral procedure used for the unexposed and PSS-exposed rats. Vertical open arrows represent i.p. injection (sertraline or saline). (a) Prevalence of EBR rats. (b) Prevalence of MBR rats. (c) Prevalence of PBR rats. The prevalence of extreme behaviora response among predator-exposed rats injected with saline was $30 \%$ of the total population, while the prevalence of MBR was $7 \%$ of the total population. Injection of sertraline immediately after the stressogenic event significantly diminished the prevalence of EBR rats to $17 \%$. IC = immediate (saline) control, IS = immediate sertraline.

mean startle amplitude (Figure 5a), as compared to unexposed controls (post hoc Bonferroni test: $t=5.3$, $p<0.0001)$. Rats given the DS treatment regimen showed a relative decrease in mean startle amplitude, as compared 

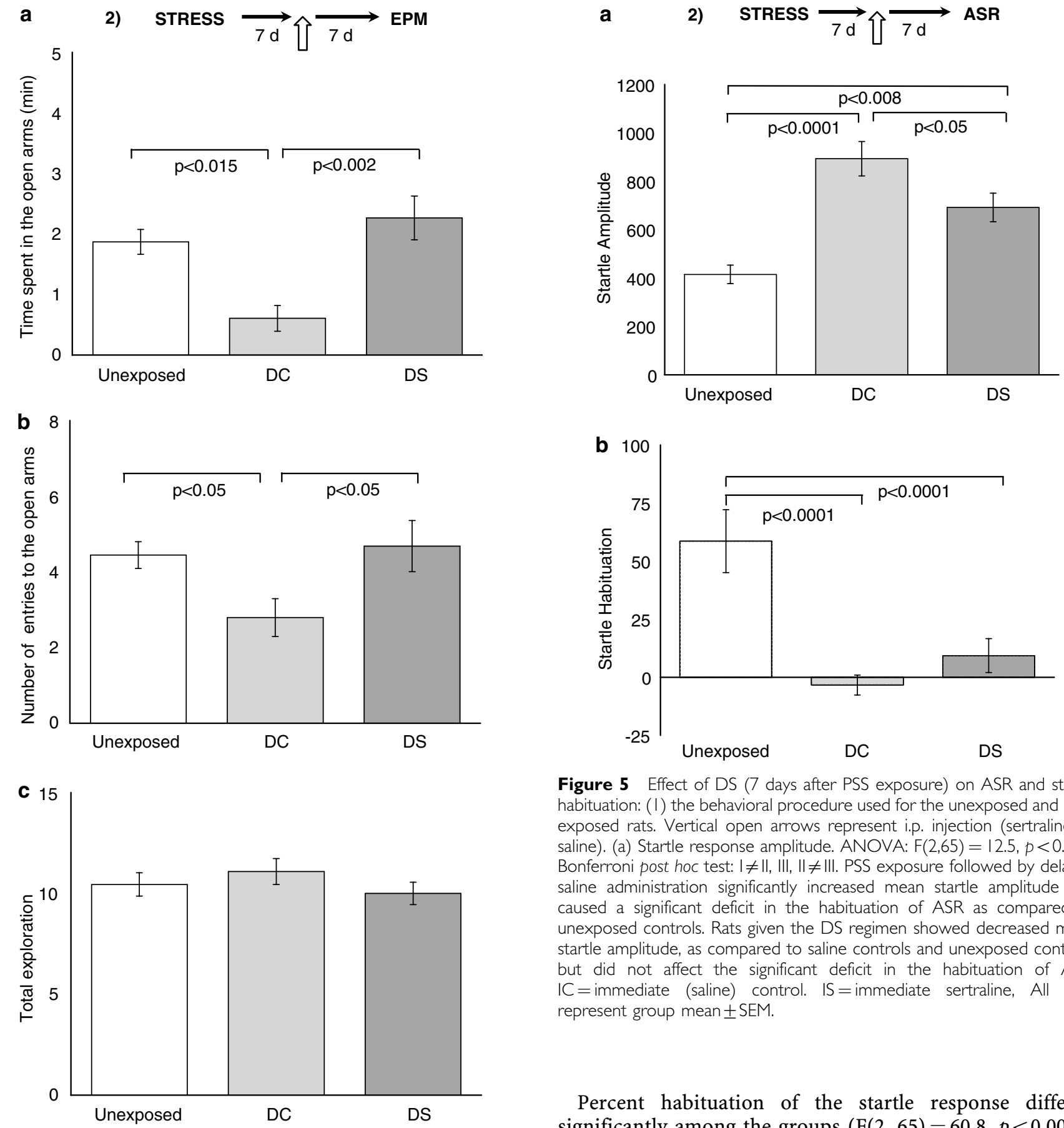

Figure 5 Effect of DS (7 days after PSS exposure) on ASR and startle habituation: (I) the behavioral procedure used for the unexposed and PSSexposed rats. Vertical open arrows represent i.p. injection (sertraline or saline). (a) Startle response amplitude. ANOVA: $F(2,65)=12.5, p<0.001$, Bonferroni post hoc test: $I \neq \|$, III, $I|\neq|||$. PSS exposure followed by delayed saline administration significantly increased mean startle amplitude and caused a significant deficit in the habituation of ASR as compared to unexposed controls. Rats given the DS regimen showed decreased mean startle amplitude, as compared to saline controls and unexposed controls, but did not affect the significant deficit in the habituation of ASR. $I C=$ immediate (saline) control. $I S=$ immediate sertraline, All data represent group mean \pm SEM.

Percent habituation of the startle response differed significantly among the groups $(\mathrm{F}(2,65)=60.8, p<0.0001)$ (Figure $5 b$ ). In both the saline and the sertraline treatment groups, stress exposure caused a significant deficit in the habituation of ASR in the exposed animals compared with naïve unexposed rats (Bonferroni test: $t=10.6, p<0.0001$ for saline-treated rats; and $t=8.6, p<0.0001$ for sertralinetreated animals). The sertraline treatment did not significantly affect habituation.

\section{Reanalysis Applying CBC}

There were significant differences in the prevalence of EBR individuals among groups (Pearson $\chi^{2}=15.7, \mathrm{df}=2$, $p<0.0004)$. As shown in Figure $6 \mathrm{a}$, in the unexposed controls there were no EBR individuals. The prevalence of 
EBR rats in the exposed group given the delayed saline regimen was $25 \%$, significantly different from unexposed controls $\left(\chi^{2}=5.25, p<0.025\right)$. The DS regimen reduced this number to $19.2 \%$ of the total population. This difference was significant compared to unexposed controls $\left(\chi^{2}=3.9\right.$, $p<0.05)$, but not significant compared to saline-treated controls.
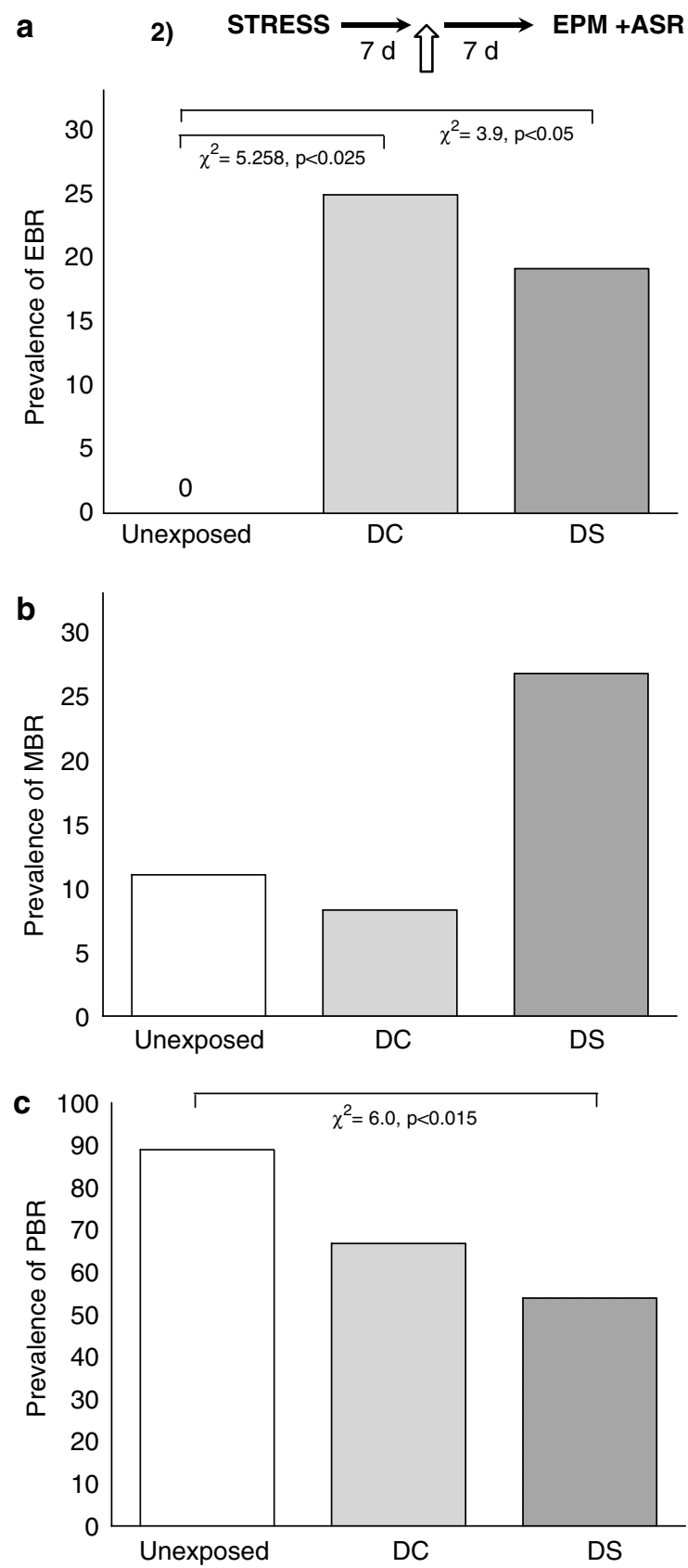

Figure 6 Effect of DS regimen on the prevalence of EBR, PBR, and MBR animals: (I) the behavioral procedure used for the unexposed and PSSexposed rats. Vertical open arrows represent i.p. injection (sertraline or saline). (a) Prevalence of EBR rats. (b) Prevalence of MBR rats. (c) Prevalence of PBR rats. The prevalence of EBR rats among the salineinjected group was $24 \%$, while the prevalence of MBR was $8.3 \%$. Delayed injection of sertraline reduced this number to a $20 \%$ and increased the proportion of MBR rats to $27 \%$ of the total population. IC =immediate (saline) control. IS = immediate sertraline.
As shown in Figure 6b, there were no significant differences in the prevalence of minimally affected individuals (MBR) among groups, although the DS group demonstrated a marked, but not significant increase (27\%).

As shown in Figure $6 \mathrm{c}$, there were significant differences in the prevalence of individuals displaying PBR among groups (Pearson $\chi^{2}=5.99, \mathrm{df}=2, p<0.05$ ). The unexposed group displayed $90 \%$ PBR. The prevalence of PBR rats in the exposed group given the delayed saline regimen was $66 \%$, not significantly different from rates for unexposed controls or the DS regimen group. The DS regimen diminished the prevalence of PBR rats to $50 \%$ of the total population, a significant effect as compared to controls $\left(\left(\chi^{2}=6.0\right.\right.$, $p<0.015)$.

\section{DISCUSSION}

This study shows that a brief immediate postexposure intervention with sertraline has an observable short-term effect on stress-induced behavioral changes in an animal model, compared to a saline-treated control group comparable the same treatment regimen at a later stage in the course of symptom development.

Seven days of treatment with sertraline immediately after PSS exposure elicited a statistically significant reduction of $14 \%$ in prevalence rates of individuals fulfilling criteria for extreme response (EBR) (and an increase of 5\% in prevalence rates of minimal response (MBR)) compared to the placebo-control group. The animals displayed a reduced prevalence of extreme anxiety-like and avoidant behaviors on the EPM and an attenuation of the exaggerated hyperarousal responses, equivalent to the patterns of behavior of unexposed animals, and a significant degree of reversal of the deficit in habituation of the ASR.

The comparison group, which was intended to model treatment of the established syndrome and was given the same treatment regimen initiated at a time which has been demonstrated to represent the cutoff point between temporary responses and the establishment of long-term, stable chronic PTSD-like behavioral changes in rats in a series of studies (Cohen and Zohar, 2004; Cohen et al, 2004), that is, 7 days after exposure, displayed a different pattern of response. The observed short-term effects on anxiety-like and avoidance behaviors on the EPM and in amplitude of ASR indicate that the delayed regimen had an alleviating effect. There was, however, no effect on startle habituation, in contrast with the marked effect of the IS regimen. The clinical implications of this difference are uncertain, but this parameter is integral to the behavioral cutoff criteria, which have been repeatedly validated.

In consequence, the prevalence rates of EBR individuals demonstrated a minor reduction (19.5\%) compared to saline controls, whereas the trend towards an increased in MBR rats was stronger (27\%), although not statistically significant. Thus, although the brief delayed treatment regimen had an effect on stress-related behavioral changes, the same brief regimen administrated immediately after exposure significantly reduced prevalence rates of extreme behavioral responses.

The brevity of the treatment regimens and the follow-up period preclude a quantitative comparison between the two 
regimens, but do confirm that immediate intervention has at least a qualitatively similar effect to the delayed regimen.

The literature contains only sparse information regarding drug interventions in the acute phase of the response to traumatic stress. Whereas treatment guidelines for acute and chronic PTSD are founded on extensive empirical evidence, in the first month following exposure to stress psychosocial treatments have predominated research (Ehlers and Clark, 2003). As for drug interventions, clinical studies have shown that early treatment with benzodiazepines in fact were ineffective in preventing PTSD in the long-term in double-blind, placebo-controlled studies (Asnis et al, 2004; Davidson, 2004b; Gelpin et al, 1996; Sansone et al, 2003), whereas intervention with $\beta$-blockers appears to have effects on the memory-consolidation process, above and beyond any symptomatic relief they may offer in terms of the physical manifestations of anxiety (Pitman et al, 2002).

The marked reduction in prevalence of extremely disrupted PTSD-like behavioral responses to PSS exposure raises the possibility that, like the $\beta$-blockers mentioned above, SSRI's may attenuate the more severe clinical sequelae of stress exposure when administered soon after exposure. Such effects might be mediated by qualities such as the following:

(1) Nonspecific effects on other neuroactive monoamines: although the SSRI antidepressants are believed to act mainly by selectively binding to the 5-HT transporter to block reuptake of 5-HT from the synapse into the presynaptic nerve terminal, thereby increasing synaptic serotonin concentrations, some of the SSRIs also exhibit other neuropharmacologic effects, such as partial norepinephrine reuptake inhibition (paroxetine) (Cryan et al, 2004; Owens et al, 2000), partial dopamine reuptake inhibition (sertraline) (Cryan et al, 2004) and reduced activity of CRF neurons (Nemeroff et al, 2006; Nemeroff and Owens, 2004). The SSRI paroxetine was found to reverse CRF alterations induced by stress associated with early maternal separation in primates (Nemeroff and Owens, 2004).

(2) Neuroprotective effects: In addition to modulating synaptic function in the adult brain, and regulating neurite outgrowth, synaptogenesis and cell survival (Mattson et al, 2004), 5-HT controls important functions in hippocampal neurogenesis (Santarelli et al, 2003). Serotonin can also promote the survival of neurons in the adult brain, as demonstrated by the abilities of a 5-HT receptor agonist and an SSRI to protect neurons against excitotoxicity and ischemic injury in animal models (Semkova et al, 1998).

(3) Neurotrophic factors: increases in neurotrophic factors, particularly brain-derived neurotrophic factor (BDNF), have been proposed to be critical for the action of antidepressants in the hippocampus (Duman, 1998; Duman et al, 1997; Duman et al, 2000; Manji and Duman, 2001). BDNF, is a survival-promoting molecule, known to modulate synaptic plasticity and neurotransmitter release across multiple neurotransmitter systems, and is involved in intracellular signal-transduction pathways (Hyman et al, 1991; Maisonpierre et al, 1991). It influences the growth and plasticity of serotonergic neurons via the activation of trkB receptor. Stress results in a downregulation of BDNF mRNA in the hippocampus, an effect which may be partially related to glucocorticoid release (Smith et al, 1995), an effect blocked by SSRI treatment (Duman et al, 1997; Nibuya et al, 1995). Interestingly, endogenous BDNF is integral to the memory consolidation process (Lee et al, 2004) through dual effects on postsynaptic gene expression and local protein synthesis (Bramham and Messaoudi, 2005). Lower levels of BDNF after stress exposure may impair the consolidation process, attenuating the anomalous traumatic memory consolidation process thought to occur in PTSD patients.

Extensive long-term studies are needed to directly assess the relevance of any of these hypothetical mechanisms to the behavioral changes seen in this preliminary study. The present study paves the way for more extensive studies of these drugs as agents of secondary prevention in the acute postexposure phase. Longer-term follow-up studies are needed to assess the effect of brief treatment regimens $v s$ long-term administration on chronic symptom/behavior patterns.

\section{Conclusions}

Brief immediate postexposure administration of an FDAapproved drug treatment for PTSD - sertraline - reduced the prevalence rates of individuals displaying extremely disrupted behaviors. These finding suggest that SSRIs drugs represent potential agents for secondary intervention in the acute aftermath of traumatic stress exposure and are thus worthy of further investigation.

\section{ACKNOWLEDGEMENTS}

This study was made possible by a research grant to ZJ and CH from Pfizer Research Department New York, USA.

\section{REFERENCES}

Adamec R (1997). Transmitter systems involved in neural plasticity underlying increased anxiety and defense-implications for understanding anxiety following traumatic stress. Neurosci Biobehav Rev 21: 755-765.

Adamec RE, Blundell J, Burton P (2003). Phosphorylated cyclic AMP response element binding protein expression induced in the periaqueductal gray by predator stress: its relationship to the stress experience, behavior and limbic neural plasticity. Prog Neuropsychopharmacol Biol Psychiatry 27: 1243-1267.

Adamec RE, Shallow T (1993). Lasting effects on rodent anxiety of a single exposure to a cat. Physiol Behav 54: 101-109.

Asnis GM, Kohn SR, Henderson M, Brown NL (2004). SSRIs versus non-SSRIs in post-traumatic stress disorder: an update with recommendations. Drugs 64: 383-404.

Bramham CR, Messaoudi E (2005). BDNF function in adult synaptic plasticity: the synaptic consolidation hypothesis. Prog Neurobiol 76: 99-125.

Bryant RA (2005). Predicting posttraumatic stress disorder from acute reactions. J Trauma Dissociation 6: 5-15.

Cohen H, Joseph Z, Matar M (2003). The Relevance of differential response to trauma in an animal model of post-traumatic stress disorder. Biol Psychiatry 53: 463-473. 
Cohen H, Kaplan Z, Matar A, Loewenthal U, Kozlovsky N, Zohar J (2006a). Anisomycin, a protein synthesis inhibitor, disrupts traumatic memory consolidation and attenuates post traumatic stress response in rats. Biolo Psychiatry (in press).

Cohen H, Zohar J (2004). Animal models of post traumatic stress disorder: the use of cut off behavioral criteria. Ann NY Acad Sci 1032: 167-178.

Cohen H, Zohar J, Gidron Y, Matar M, Belkind D, Loewenthal U et al (2006b). Blunted HPA axis response to stress influences susceptibility to posttraumatic stress response in rats. Biol Psychiatry (in press).

Cohen H, Zohar J, Matar MA, Kaplan Z, Geva AB (2005). Unsupervised Fuzzy Clustering Analysis Supports Behavioral Cutoff Criteria in an Animal Model of Posttraumatic Stress Disorder. Biol Psychiatry 58: 640-651.

Cohen H, Zohar J, Matar MA, Zeev K, Loewenthal U, Richter-Levin G (2004). Setting apart the affected: the use of behavioral criteria in animal models of post traumatic stress disorder. Neuropsychopharmacology 29: 1962-1970.

Cryan JF, O'Leary OF, Jin SH, Friedland JC, Ouyang M, Hirsch BR et al (2004). Norepinephrine-deficient mice lack responses to antidepressant drugs, including selective serotonin reuptake inhibitors. Proc Natl Acad Sci USA 101: 8186-8191; E-pub 2004 May 17.

Davidson JR (2003). Treatment of posttraumatic stress disorder: the impact of paroxetine. Psychopharmacol Bull 37: 76-88.

Davidson JR (2004a). Remission in post-traumatic stress disorder (PTSD): effects of sertraline as assessed by the Davidson Trauma Scale, Clinical Global Impressions and the Clinician-Administered PTSD scale. Int Clin Psychopharmacol 19: 85-87.

Davidson JR (2004b). Use of benzodiazepines in social anxiety disorder, generalized anxiety disorder, and posttraumatic stress disorder. J Clin Psychiatry 65: 29-33.

Duman RS (1998). Novel therapeutic approaches beyond the serotonin receptor. Biol Psychiatry 44: 324-335.

Duman RS, Heninger GR, Nestler EJ (1997). A molecular and cellular theory of depression. Arch Gen Psychiatry 54: 597-606.

Duman RS, Malberg J, Nakagawa S, D'Sa C (2000). Neuronal plasticity and survival in mood disorders. Biol Psychiatry 48: 732-739.

Ehlers A, Clark D (2003). Early psychological interventions for adult survivors of trauma: a review. Biol Psychiatry 53: 817-826.

File SE (1993). The interplay of learning and anxiety in the elevated plus-maze. Behav Brain Res 58: 199-202.

Foa EB, Cahill SP, Boscarino JA, Hobfoll SE, Lahad M, McNally RJ et al (2005). Social, psychological, and psychiatric interventions following terrorist attacks: recommendations for practice and research. Neuropsychopharmacology 30: 1806-1817.

Gelpin E, Bonne O, Peri T, Brandes D, Shalev AY (1996). Treatment of recent trauma survivors with benzodiazepines: a prospective study. J Clin Psychiatry 57: 390-394.

Hyman C, Hofer M, Barde YA, Juhasz M, Yancopoulos GD, Squinto $\mathrm{SP}$ et al (1991). BDNF is a neurotrophic factor for dopaminergic neurons of the substantia nigra. Nature 350: 230-232.

Kim SW, Park SY, Hwang O (2002). Up-regulation of tryptophan hydroxylase expression and serotonin synthesis by sertraline. Mol Pharmacol 61: 778-785.

Lee JL, Everitt BJ, Thomas KL (2004). Independent cellular processes for hippocampal memory consolidation and reconsolidation. Science 304: 839-843; E-pub 2004 April 8.

Maisonpierre PC, Le Beau MM, Espinosa 3rd R, Ip NY, Belluscio L, de la Monte SM et al (1991). Human and rat brain-derived neurotrophic factor and neurotrophin-3: gene structures, distributions, and chromosomal localizations. Genomics 10: 558-568.

Maj J, Rogoz Z (1999). Synergistic effect of pramipexole and sertraline in the forced swimming test. Pol J Pharmacol 51: 471-475.
Manji HK, Duman RS (2001). Impairments of neuroplasticity and cellular resilience in severe mood disorders: implications for the development of novel therapeutics. Psychopharmacol Bull 35: 5-49.

Marona-Lewicka D, Nichols DE (1997). The effect of selective serotonin releasing agents in the chronic mild stress model of depression in rats. Stress 2: 91-100.

Mattson MP, Maudsley S, Martin B (2004). BDNF and 5-HT: a dynamic duo in age-related neuronal plasticity and neurodegenerative disorders. Trends Neurosci 27: 589-594.

Nemeroff CB, Bremner JD, Foa EB, Mayberg HS, North CS, Stein MB (2006). Posttraumatic stress disorder: a state-of-the-science review. J Psychiatr Res 40: 1-21; E-pub 2005 October 18.

Nemeroff CB, Owens MJ (2004). Pharmacologic differences among the SSRIs: focus on monoamine transporters and the HPA axis. CNS Spectr 9: 23-31.

Nibuya M, Morinobu S, Duman RS (1995). Regulation of BDNF and trkB mRNA in rat brain by chronic electroconvulsive seizure and antidepressant drug treatments. J Neurosci 15: 7539-7547.

Owens MJ, Knight DL, Nemeroff CB (2000). Paroxetine binding to the rat norepinephrine transporter in vivo. Biol Psychiatry 47: 842-845.

Pitman RK, Delahanty DL (2005). Conceptually driven pharmacologic approaches to acute trauma. CNS Spectr 10: 99-106.

Pitman RK, Sanders KM, Zusman RM, Healy AR, Cheema F, Lasko $\mathrm{NB}$ et al (2002). Pilot study of secondary prevention of posttraumatic stress disorder with propranolol. Biol Psychiatry 51: 189-192.

Sansone RA, Hruschka J, Vasudevan A, Miller SN (2003). Benzodiazepine exposure and history of trauma. Psychosomatics 44: 523-524.

Santarelli L, Saxe M, Gross C, Surget A, Battaglia F, Dulawa S et al (2003). Requirement of hippocampal neurogenesis for the behavioral effects of antidepressants. Science 301: 805-809.

Semkova I, Wolz P, Krieglstein J (1998). Neuroprotective effect of 5-HT1A receptor agonist, Bay X 3702, demonstrated in vitro and in vivo. Eur J Pharmacol 359: 251-260.

Shalev AY (2002). Acute stress reactions in adults. Biol Psychiatry 51: 532-543.

Smith MA, Makino S, Kvetnansky R, Post RM (1995). Stress and glucocorticoids affect the expression of brain-derived neurotrophic factor and neurotrophin-3 mRNAs in the hippocampus. J Neurosci 15: 1768-1777.

Stein DJ, Bandelow B, Hollander E, Nutt DJ, Okasha A, Pollack MH et al (2003). WCA Recommendations for the long-term treatment of posttraumatic stress disorder. CNS Spectr 8: 31-39.

Thomas DN, Nutt DJ, Holman RB (1998). Sertraline, a selective serotonin reuptake inhibitor modulates extracellular noradrenaline in the rat frontal cortex. J Psychopharmacol 12: 366-370.

Tucker P, Ruwe WD, Masters B, Parker DE, Hossain A, Trautman $\mathrm{RP}$ et al (2004). Neuroimmune and cortisol changes in selective serotonin reuptake inhibitor and placebo treatment of chronic posttraumatic stress disorder. Biol Psychiatry 56: 121-128.

Ursano RJ, Bell C, Eth S, Friedman M, Norwood A, Pfefferbaum B et al (2004). Practice guideline for the treatment of patients with acute stress disorder and posttraumatic stress disorder. $A m \mathrm{~J}$ Psychiatry 161: 3-31.

Weine S, Danieli Y, Silove D, Van Ommeren M, Fairbank JA, Saul J (2002). Guidelines for international training in mental health and psychosocial interventions for trauma exposed populations in clinical and community settings. Psychiatry 65: 156-164.

Zohar J, Amital D, Miodownik C, Kotler M, Bleich A, Lane RM et al (2002). Double-blind placebo-controlled pilot study of sertraline in military veterans with posttraumatic stress disorder. J Clin Psychopharmacol 22: 190-195. 